\title{
A Property-Rights Mismatch Approach to Passive- Active Spectrum Use Coexistence
}

\author{
Martin BH Weiss $\pm^{*,+1}$ Ali Palida $^{+}$, Ilia Murtazashvili ${ }^{+}$, \\ Prashant Krishnamurthy* \\ *Dept of Informatics \& Networked Systems \\ ${ }^{+}$Ctr for Governance \& Markets \\ University of Pittsburgh \\ Pittsburgh PA, USA \\ mbw, afp31, ilia.murtazashvili, prashk@pitt.edu
}

\author{
Philip J. Erickson \\ Haystack Observatory \\ Massachusetts Institute of Technology \\ Cambridge, MA, USA \\ pje@mit.edu
}

\begin{abstract}
Policies and regulations governing electromagnetic spectrum prioritize reducing conflict among active users of spectrum (transmitters), thereby enabling these active users to capture the values associated with property rights to spectrum. Coexistence of heterogeneous technologies and their enforcement have been well studied, but much less has been done to consider the coexistence of heterogeneous uses and the institutions that are necessary to address conflict arising among different users of spectrum.

We argue that prevailing property-rights institutions that focus on reducing conflict among active users of spectrum generate a property mismatch that contributes to conflict with passive users of spectrum. Passive users are interested primarily in receiving signals transmitted by nature. The propertymismatch approach offers insight into how to redesign spectrum governance to balance the demands of both active and passive users. Particularly we argue that virtual parceling of the electromagnetic spectrum along a broader range of dimensions can better facilitate efficient spectrum sharing between active and passive users.
\end{abstract}

Keywords-active use, passive use, property mismatch, radio spectrum, commons

\section{INTRODUCTION}

The literature on spectrum sharing overwhelmingly focuses on active uses of spectrum, especially transmitters [1]. This dates from the earliest days of radio wave development and is understandable since active uses have direct economic impacts, such as media transmission via television and radio broadcasts and societal communication benefits offered by $\mathrm{Wi}-\mathrm{Fi}$ and mobile phone services. The prevailing approach to spectrum management involves licensing spectrum with attendant prescriptions on emitter characteristics, or, in certain instances, allowing unlicensed use (e.g., US FCC Part 15 systems).
However, another important application of spectrum involves passive use of electromagnetic spectrum. In passive use, the "transmitters" are natural phenomena, whose time, frequency, and strength are (usually) outside of human control due to the properties of matter and energy. These passive uses are intermediate products with indirect economic impacts and have traditionally received less policy consideration than active uses, the latter of which have been subject to substantial regulatory consideration since the $1920 \mathrm{~s}$ in the US. Some notable passive scientific uses include radio astronomy and Earth remote sensing including meteorological and upper atmosphere applications. Passive use cases may involve terrestrial or space-based receiving antennas and, in a large majority of cases, must be capable of detecting extremely low energy signals, bound to the frequencies in which the phenomena of interest must be observed. These can be at or below the thermal noise floor set by cosmic processes and material instabilities. These passive uses offer significant social benefits, though they do not always provide direct and immediate benefits compared to active uses in which property rights confers an immediate value or a right to future values. The dominant governance institutions for radio spectrum have generally been more oriented to the demands of active users, and establishing property rights that reflect those demands, as opposed to passive users.

In this paper, we show how the regulatory institutions and policies that emerged primarily in response to concerns about management of active users contribute to conflict in radio spectrum with passive users. We propose the property-mismatch approach as a general framework for analyzing conflict over the radio spectrum [2], [3]. Conventional property analysis considers electromagnetic spectrum as a congestible commons [4]. According to this view, a central consideration for public policy is to better align property rights so that users can capture

\footnotetext{
${ }^{1}$ Author for Correspondence.
} 
the value from the scare resource [5], [6]. The propertymismatch approach extends the conventional resource commons approach by considering how the underlying resource (in this case spectrum) may operate at different scales than the prevailing property regime. Hence, it provides a useful framework for addressing and, we argue, for resolving such conflicts.

We highlight that the current liberal licensing regime designed for managing conflict among active users, through exclusive and tradeable rights to spectrum frequencies, does not adequately address the temporal, spatial, quantity-, and qualitybased mismatches between active and passive users of spectrum. Furthermore, we show that the property-mismatch approach has offered insight into the design of institutions to resolve disputes over natural resources. When applied to spectrum, this approach has significant implications for reforms to balance the interests of active and passive users of spectrum. Particularly, we propose that finer virtual parceling of the electromagnetic spectrum along the dimensions of time, frequency, and transmission power can better facilitate efficient spectrum sharing between active and passive users.

The property-mismatch approach we suggest is a significant change in in perspective in that it recognizes limitations with conventional property rights analysis of spectrum, while simultaneously recognizing spectrum has many characteristics of a commons. Some research contends that spectrum is not a commons [4], and that the prevailing system of licensing contributes to inefficiencies in allocation of spectrum [5]. We instead see spectrum as a commons, and further assert that the property regime - not the inherent nature of the resources - can contribute to unwanted conflict over spectrum.

\section{PROPERTY RIGHTS IN SPECTRUM}

In the context of resource commons, property rights are conventionally conceptualized as a bundle of rights with the following dimensions: (1) access (right to entry); (2) withdrawal (right to extraction); (3) management (right to regulate and make improvement); (4) exclusion (right to determine access and transfer; and (5) alienation (right to sell or lease management and exclusion rights) [9]. Weiss et.al. [3] applied this to spectrum by associating access rights with the right to receive and the withdrawal rights as the right to transmit. Management and exclusion rights are dependent on the allocation and license terms, and alienation rights do not apply to unlicensed spectrum.

The conventional approach to radio spectrum management involves licensing radio transmitters. Licensing can be divided into two broad approaches: traditional and liberal licenses. In contrast, allocating unlicensed spectrum bands has also recently been implemented, and this model provides rich opportunities for self-governance of spectrum usage.

\section{A. Traditional Licenses}

Under traditional licensing, radio spectrum is nationalized as an economic asset, and use rights are allocated. Traditional licensing consists of two phases: allocation and assignment. Allocation specifies permitted uses of a band (and often occurs at the international level) and assignment specifies who is granted the operating license on which band (or sub-band) in which location for which times. Additional technical restrictions on the nature of the emitter, such as required out-ofband emission masks, are applied at time of assignment.

Traditional licensing is based on allocation through an administrative process, with rights assigned based on political considerations such as "public interest" [1]. Furthermore, traditional licenses can impose additional geographic, temporal and frequency constraints.

\section{FEATURES}

\begin{tabular}{cl}
\hline TRADITIONAL & $*$ Transmission rights assigned on a \\
LICENSES & time/space/frequency basis \\
& $*$ License allocated for intended use \& \\
& (sometimes) "public interest" \\
LIBERAL & $*$ Transmission rights assigned on a \\
LICENSES & time/space/frequency basis \\
& $*$ Alienation rights \\
& $*$ Flexible uses (allocation) \\
UNASSIGNED & $*$ Allocated but not assigned \\
& $*$ May be unlicensed \\
& $*$ Properties of a commons \\
& $*$ Transmission power caps \\
& $*$ Etiquette
\end{tabular}

Table 1. Property regimes governing active users

\section{B. Liberal Licenses}

Like traditional licensing, liberal licensing involves a nationalized spectrum with rights distributed to users in allocation and assignment phases. Unlike traditional licensing, the liberal licensing regime involves re-allocation of property rights to spectrum through competitive markets [7].

Because of the growing diversity of uses, innovations in radio technologies, and inefficiencies in the traditional allocation approach, an alternative regime has emerged in some countries and some frequency bands. In this approach, licenses are treated as quasi-property. Licenses can be bought and sold on a secondary market and license owners have greater flexibility of use, as long as they remain within the transmission power parameters of the license.

\section{Unassigned}

A yet different approach to spectrum use is one that eschews the need for specific assignments entirely. In this approach, transmission bands are allocated to a use but not assigned to a particular user in a particular time/space/frequency tuple. The two most common examples of this are unlicensed bands and amateur radio bands. These unassigned bands have characteristics (and challenges) similar to passive use, especially some amateur bands where long distance propagation is possible.

Here, maximum transmission power is defined as before (though usually at a lower level) and a spectrum etiquette may be established for anyone who seeks to use this band. These community norms may be behavioral rules that are enforced by the community, or they may be embedded in the hardware/software of devices using these bands. These unassigned bands have many of the economic characteristics of a commons [8]. Experience has shown that these bands have 
been a fruitful resource for innovation for systems that use wireless communications because of the relative ease of obtaining spectrum. Table 1 summarizes the key features of property regimes governing active users.

\section{Mismatches In ACTIVE AND PASSIVE SPECTRUM USE}

Property rights mismatch occurs when the bundle of rights to achieve certain ends results in conflict or missed opportunities for exchange. Yandle [2] defines property mismatch as "a problem of fit involving human institutions that do not map coherently on the biogeophysical scale of the resource either in time or space..." The property rights mismatch approach adds to the previous list of five features of property regimes the following: (6) temporal (period for which operational-level rights may be exercised); and (8) quantitative (how much of a resource may be extracted in a given period) [2], [10]. In addition, we include a category for quality (9), which as we explain below considers in the differences in sensitivity of the radio receiving equipment. Signal quality (such as signal to noise or interference ratios) in the digital world impacts data rates and error rates. Since emitters are under human control, there are ways to tradeoff error rates with coding or signal processing. In typical passive usage cases, such tradeoffs are not possible, and in addition, the receiver must be sensitive enough to detect extremely small energy levels.

In this context, since passive users do not transmit, they do not fit the operating model behind the license approach. Instead of a transmit power, passive users are more interested in minimizing electromagnetic energy from active sources in the frequency of interest at their antenna terminals. This strategy has been traditionally difficult to regulate, since the mathematical and physical transform mapping transmitter to receiver is a high dimensionality object with many unknown characteristics driven by fundamental physics and significant spatio-temporal variability. For instance, electromagnetic propagation can best be modelled as a random process with incompletely known independent variables even in a statistical sense, and with a strong influence from background atmospheric and ionospheric conditions.

History has shown that traditional licenses have not been a good fit for passive users. Because of this, the passive community has sought to exercise their influence in the allocation phase of spectrum management: passive users have sought to have bands set aside for these uses, so no licenses can be assigned to active users in these bands (i.e., a mutual exclusion approach).

Allocations have not had a property right association as assignments have had. Allocations are not auctioned; instead, they are determined through a deliberative process. None of the traditional spectrum management organizations have had a responsibility for enforcing the "boundaries" of an allocation in the way that has been the case for assignments. Thus one source of mismatch is the differing locus of rights definition.

However, as pointed out by the National Research Council [9], the increasing active uses of radio spectrum are creating challenges to the passive users from the increased (unintentional) interference from active uses and from the increased sensitivity of the instruments used for passive users. Using the language of the mismatch literature on resources, governance of the property rights for passive users is more akin to considerations at the level of a landscape, rather than an individual parcel (or exclusive license).

This mismatch also extends to the enforcement of rights. Demsetz [18] argued that enforcement is a critical part of any property regime. This has been confirmed by Ostrom [10], [11] in her analysis of common pool resource governance regimes. In addition to ensuring the value of property rights, a predictable enforcement system fosters trust between transacting parties, which reduces transaction costs. For active users, enforcement consists of detecting the presence and location of offending transmitters leading to remediation, which normally consists of a "cease and desist" order. In the US, enforcement may be carried out privately by license holders or by the FCC's Enforcement Bureau [12]. For passive users, however, enforcement is challenging because they do not have an inherent property right in the form of a license. Enforcement of allocations is much weaker and many passive users do not have the resources to engage in private enforcement activities since they are overwhelmingly not-for-profit entities or government agencies. As well, it is often the case that interference to passive uses comes from adjacent frequency bands and may be caused by poor design, build, or maintenance of the interfering devices, making enforcement even more costly.

\section{A. Dimensions of Rights Mismatch}

Mitigating interference is possible in some limited cases through careful frequency allocation and other technological uses. However, because the scale of the interference problems are quite different, the mismatched property rights theory provides a useful analytical framework. In particular, active radio systems operate at different scales from passive users, on a number of important dimensions (summarized in Table 2).

Temporal - The frequencies needed for passive uses are dictated by the phenomena and physical medium properties being studied. Unlike active users, the frequency "allocations" are typically stable and permanent and do not vary with demand. (Of course, the medium state can change these allocations, but their fundamental spectral allocations remain tied to physical properties.) The particular moments (or intervals) when passive users' observations must take place are dictated by the phenomenon under study and the available technology ${ }^{2}$. By contrast, the demand for active use is largely determined by the demand for the services provided on a spatio-temporal basis.

Spatial - Active users often have licenses (hence operations) that may be local, regional and sometimes national in scope. However, passive remote sensing applications can span hundreds of kilometers or even up to and beyond the planet's diameter. Furthermore, passive remote sensing is often transnational, and passive users may be sensitive to interference from multiple legal jurisdictions.

\footnotetext{
${ }^{2}$ For instance, some observations are taken over long time periods to increase the sensitivity of the measurement.
} 
Quantity - Active users' demand for spectrum is strongly correlated with demand for their service. In contrast, passive users are often driven by the unpredictable nature of scientific discovery.

Quality - Passive users in nearly all cases require receivers that are much more sensitive than those used for active use. This is the case because passive users do not control the strength of the emissions they are observing (as they result from natural phenomena) unlike active users.

\section{PASSIVE}

\begin{tabular}{|c|c|c|}
\hline TEMPORAL & $\begin{array}{l}\text { Frequency } \\
\text { bands/time } \\
\text { determined by } \\
\text { phenomena. } \\
\text { Frequency } \\
\text { requirements } \\
\text { extremely stable. }\end{array}$ & $\begin{array}{l}\text { Frequency bands } \\
\text { and time } \\
\text { determined by } \\
\text { business model or } \\
\text { customer demand } \\
\text { and may be } \\
\text { dynamic in time, } \\
\text { space and } \\
\text { frequency band. }\end{array}$ \\
\hline SPATIAL & $\begin{array}{l}\text { Determined by } \\
\text { required maximum } \\
\text { interference level; } \\
\text { Frequency } \\
\text { allocation needs } \\
\text { may cross license or } \\
\text { jurisdictional } \\
\text { boundaries. }\end{array}$ & $\begin{array}{l}\text { Determined by } \\
\text { license (e.g., on a } \\
\text { an MSA, basis), } \\
\text { regulation (power } \\
\text { caps) or } \\
\text { propagation } \\
\text { characteristics }\end{array}$ \\
\hline QUANTITY & $\begin{array}{l}\text { Required frequency } \\
\text { bands extremely } \\
\text { stable; determined } \\
\text { by material and } \\
\text { energy properties. }\end{array}$ & $\begin{array}{l}\text { Increasing } \\
\text { requirements in } \\
\text { time, frequency } \\
\text { and space to meet } \\
\text { customer demand }\end{array}$ \\
\hline QUALITY & $\begin{array}{l}\text { Often involves very } \\
\text { low Signal to Noise } \\
\text { ratios. Very low } \\
\text { noise and } \\
\text { interference } \\
\text { tolerance }\end{array}$ & $\begin{array}{l}\text { Signal quality and } \\
\text { bandwidth } \\
\text { requirements vary, } \\
\text { depending on the } \\
\text { technology used } \\
\text { and the service } \\
\text { provided. }\end{array}$ \\
\hline
\end{tabular}

Table 2: Passive/Active mismatch

\section{B. Institutional needs for Resolving Mismatches}

The property-mismatch approach suggests the necessity for governance institutions that are able to resolve the mismatch dimensions that are summarized in Table 2. To achieve the low interference and noise environments needed for passive service, a large geographic area (protection zone) is defined to reduce the co-channel interference at the passive receiver. This protection zone may overlap multiple license areas for active users. As well, passive users must seek to reduce other interference such as adjacent channel interference and harmonic interference from more spectrally distant bands. Methods to resolve this mismatch are illustrated by some specific use cases:

- At the National Radio Astronomy Observatory (NRAO), the passive/active mismatch was resolved in 1959 through the establishment of the National Radio Quiet Zone (NRQZ) in West Virginia, in which many transmissions (e.g., mobile phone, WiFi) are prohibited. This was achieved through regulatory action at the FCC. Explicit coordination procedures are described, as are specific quality requirements. With the passage of time, technology is causing this arrangement to break down. For example, inexpensive and ubiquitous radiators, such as automotive radars, are costly (or near impossible) to control, resulting in high levels of noise to NRAO operations.

- Haystack Observatory, operated by Massachusetts Institute of Technology, is a multidisciplinary radio and radar passive and active remote sensing observatory located in the Boston metro area. Haystack's proximity to a large metropolitan area, sited on top of a hill with no immediate terrain blockage, causes sensors on site to experience interference from many sources. Initially, interference protection came from exclusive licensed bands, as well as operations at multiple $10 \mathrm{~s}$ of $\mathrm{GHz}$ that were previously of no commercial interest. However, mobile radiators (such as automotive radars) and technological changes have made $\mathrm{cm}$ and $\mathrm{mm}$ wave bands commercially viable, and this has fundamentally changed the environment. As well, spurious emissions (or harmonics from other licensed bands) from large distances (e.g. within $300+\mathrm{km}$ radius for UHF ionospheric remote sensing radar) can cause severe interference to the very sensitive receivers on site in highly variable ways that are very difficult to mitigate. Environmental changes at meter wavelengths and longer have also become extremely challenging for remote sensing because of unintentionally radiated public emissions from consumer technology (e.g. widely available LED light bulbs) lacking proper type checking and RF filtering.

\section{Analysis of Current Instituions}

Clearly, these parcels can be seen as "landscape level resources" in terms of their spatial dimensions ${ }^{3}$. Passive users interact with the national and international spectrum management institutions through bodies such as the US National Academies of Science, Engineering, and Medicine's Committee on Radio Frequencies (CORF). CORF submits comments on spectrum management proceedings and policies to the FCC, participates in US and international working parties within the International Telecommunications Union (ITU)), and otherwise informs stakeholders about the passive uses of radio spectrum.

This independent, peer-reviewed institution has addressed some of these rights mismatches because the frequencies needed for passive use are very stable (being determined by the phenomena under study). CORF may be able to resolve conflicts

this, the FCC developed different license classes to account for this propagation phenomena. For more details, see www.fcc.gov/media/radio/am-clear-regional-local-channels
${ }^{3}$ The FCC has dealt with landscape level resources. For example, the AM band experiences sky waves at night which allows for very long transmission distances. To accommodate 
within the scientific and radio astronomy communities, but it lacks enforcement power with regard to conflicts with the active use community, particular in the temporal and quality dimensions.

Responsibility for spectrum management in the US is split across several different agencies. The Federal Communications Commission (FCC) is responsible for the management of commercial spectrum, while the National Telecommunications and Information Administration (NTIA) is responsible for managing government spectrum. The Department of Defense maintains a significant role in the management of spectrum for military uses. Finally, the Department of State is responsible for interacting with the International Telecommunications Union (ITU) for international spectrum allocation.

Let us consider the mismatches described in Table 2 and consider how the active US agencies might address them or if there are barriers within these agencies to address the mismatches. Although we treat these mismatches independently in each paragraph, it is important to bear in mind that they are correlated with each other in ways that have not been wellquantified.

\section{1) Temporal Mismatches}

Passive users have very stable requirements over time since the phenomena they are studying are related to particular natural phenomena (which do not change). In contrast, active uses of spectrum are increasing at an impressive rate. Given the growth of the liberal licensing regime and the high value of spectrum auctions in the past decades, the FCC is under considerable pressure to auction as much spectrum as possible. At the same time, the NTIA and DoD are under pressure to find bands to share with commercial users. Thus, these agencies are not well suited to address temporal mismatches. Furthermore, because passive users do not transmit, they are a poor fit for the assignment or license regime, which is the classical approach of these agencies. The Dept of State, which is the preeminent agency for the international allocation scheme, is not equipped to resolve temporal mismatches.

On another level, some passive measurements occur over relatively long periods of time (hours), while most individual active users use spectrum for a few minutes. Even with more intensive coordination between active and passive users, these divergent timeframes may be challenging to reconcile. One option is to have periodic "Earth Hour" pauses of active use, similar to a scheduled black-out like load shedding in the electric grid. Such pauses, which need not occur on a planetary scale, constitute a virtual parceling that balances the rights of active users with passive users. It is unclear what agencies may be best suited to implement such transmission pauses and how they would be enforced.

\section{2) Spatial Mismatches}

With few exceptions, the FCC's and NTIA's spectrum assignments have a relatively small (i.e., metropolitan scale) geographic scope. For some uses, this might be implemented through transmit power caps and for others, this is defined in the license. As described above, many passive users require regional (super-metropolitan) scale, mostly to achieve their quality requirement. Passive users often receive an allocation, so no active transmitters should be on the same band. However, these spatial requirements are necessary because of interference from a variety of out-of-band sources.

The FCC and NTIA could set strict standards for out of band emissions, but they lack the staff to adequately enforce these standards. Furthermore, those standards would increase transmitter and receiver costs, which most commercial operators would oppose. Thus identifying a new or existing agency with a comparative advantage in monitoring and unauthorized emissions may be necessary to enforce this kind of institutional arrangement.

\section{3) Quantity Mismatches}

The needs of passive users are stable, changing only when new scientific phenomena are discovered that warrant investigation. As described above, the needs of active users are related to demand for the services they are supporting. It is well known that these demands are growing. As described above, the FCC, NTIA and DoD are under pressure from active users to repurpose spectrum to support growing commercial needs. Repurposing spectrum may also be an important revenue source for governments, thus creating a political incentive to do so. Since increasing quantity allocated to active uses generates conflict with passive use, including through stray emissions, an implication is that some limits on licensing in the aggregate may be necessary. Accordingly, it may be necessary for all the agencies to coordinate on some caps or limitations on spectrum licenses since the cumulative effect of licensing is to crowd out passive use.

\section{4) Quality Mismatches}

As described above, passive users require a very low noise environment since they do not control the signal strength of the sources they are studying. By contrast, active users control both the transmitter and receiver, so the quality of the transmission can be controlled more closely. The assignment approach that dominates spectrum management at the FCC and NTIA is well suited to active users but less so for passive users. Furthermore, the enforcement arms of these agencies are generally not quipped to make the kinds of measurements needed to support enforcement actions on behalf of passive users. Further expansion of the institutional framework governing receiving spectrum, including through explicit attention to monitoring interference, would appear warranted, much like how the FCC devotes attention to monitoring transmission (e.g., in the case of radio piracy). Monitoring interference with receiving is a different issue, one that would better balance the demands of heterogenous users.

\footnotetext{
${ }^{4}$ Earth Hour in March is a grassroots effort to switch off all lights in 190 countries for an hour. See www.earthhour.org/
} 


\section{PROPERTY-MISMATCH APPROACH TO SPECTRUM GOVERNANCE AND VIRTUAL PARCELING}

Governance includes spontaneous and deliberate (or designed) order [13]. Mutually beneficial exchange is conducted through the construction of contracts, where "contracting" is defined broadly to include bargaining between politicians, bureaucrats, and economic parties who have an interest in rights and their allocation [14].

Economically, the current set of marketed contracts for spectrum-usage rights (i.e., licenses) are incomplete when the needs of passive users are considered in addition to active users, as they do not resolve the temporal, spatial, quantity, and quality based mismatches described in the previous section. One way to address mismatch is to focus on unbundle property right of active users. This involves recognition that a wider set of commoditized property rights are needed to provide users with consistent and continuous answers to the following broader set of questions:

1. How long are rights of access to spectrum guaranteed, or how much time passes until renegotiation or reassignment of property rights? (temporal)

2. How well does the governance regime define locations where operational-level rights can be exercised? (spatial)

3. How much of the radio spectrum may be used in any given time interval? (quantity)

4. What are the signal-to-noise ratios (and interference) that is necessary for successful use of spectrum? (quality).

If liquid secondary markets of contracts which clearly specify these more granular property-rights considerations are created, we believe it may be possible to alleviate some of the conflicts arising from misaligned preferences between active and passive spectrum users, perhaps even achieving efficient use [15]. Such an approach can be interpreted as "radical-market thinking" [16], which emphasizes a redesign of property-rightsspecifying commodities to facilitate a wider range of uses for a particular common-pool resource.

One possible method of attaining a radical spectrum market that efficiently caters to the needs of both active and passive users, could be through finer virtual parceling of the electromagnetic spectrum. Such a perspective recognizes that there are property rights assigned to active users and a concurrent system of property rights for passive users. Currently, time and transmit power windows together with frequency bands are each routinely used to establish property rights for active users, so they function as a limited form of "parceling" in spectrum which is consistent with conventional licensing approach pioneered by Ronald Coase [17] and Theordore Herzel [5] in the 1950s. However, this "long and skinny" partition of the spectrum, we believe, can be further broken down into smaller, more marketable components. The figure below provides a graphical depiction of how a finer partition of virtual parcels can be constructed, relative to the current exclusive licensing regime:

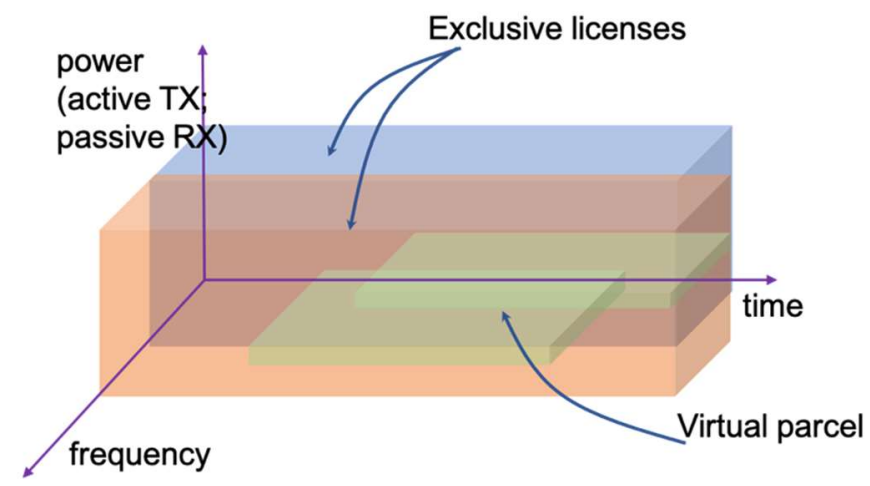

Figure 1: Virtual Parceling in Spectrum

A wide array of parcel partitions are worth considering, given current technologies which can be used to enforce and generate formal contracts specifying spectrum-usage rights. For example, out-of-band signaling can facilitate contracts specifying that active users to "back-off" when their spectrum usage generates interference with important passive phenomena. Consider a passive user of TV broadcast signals. Most TVs today are equipped with WiFi and this might be useful in signaling usage of the passive reception to avoid interference. This form monitoring can be incorporated into a virtual parcel that specifies a tolerable amount of interference from active users, after which passive users can legally exclude active users from transmitting on a particular frequency band.

Additionally, machine learning (ML) may be a useful approach to predict usage and autonomously commoditize parcels in time, frequency, and space. That is, the virtual parcels in Figure 1 may be constructed not just from human analysis of existing data on user preferences, but also from real-time predictive algorithms. ML strategies can also help deal with extracting useful, necessary, and actionable metadata from the high dimensionality hypercube formed by the radio wave propagation medium, sparsely sampled at the present in both time and space (due to the long distance nature of Coulomb forces in the Earth's magnetized environment).

We are not the first to suggest virtual parceling as a potential solution to property-rights mismatch. Bradshaw and Leonard [13] also propose "virtual parcels" to address the differing scales and dimensions of different resource types whereby "parcels" of efficient scale for the resource are identified and created through a variety of techniques (e.g., market transactions, contract, communal governance, government coordination, resource seizure through eminent domain or nationalization, etc.). Our approach is similar in that our proposal for achieving efficient spectrum allocation involves generating finer parcels of spectrum-usage rights to address temporal, spatial, quality, and quantity-based mismatches that currently occur between active and passive users under the exclusive licensing regime. Virtual parceling in the spectrum space acknowledges that there are already multiple competing rights and that through institutional design, these rights can be further unbundled in ways that more effectively balance the demands of heterogeneous users. In the process, virtual parceling contributes to more appropriate use of the electromagnetic spectrum, including by ensuring through 
institutional design continuity of the indirect benefits which accrue from passive use of spectrum.

With a broader notion of governance, what occurs is consideration not only for the substantive rules (licensing versus other), but with the processes and procedures to address conflict. In that regard, the mismatch approach provides a more substantial role for the governance institutions to address conflict, particularly the reorganization of government institutions to include a finer set of marketed parcels of spectrum-usage rights. The mismatch approach also highlights that organizational incentives may not be aligned with the most appropriate use of spectrum, such as with the FCC and related agencies having such strong incentives to further parcel out the spectrum for active uses. Currently, there is no organization whose purpose is to represent the interests of passive users. A property mismatch approach suggests that perhaps there should be, or at a minimum, that a governance organization that emerged to address the demands of active users and is under increasing pressure to further divide spectrum for active use may not be the best equipped to address spectrum conflicts.

\section{DISCUSSION AND CONCLUSION}

The current institutional framework for allocation radio spectrum was developed primarily with active users in mind, in particular transmitters. One of the challenges with the focus on active users is that passive use is often not sufficiently accounted for. There are several reasons for this, including that the property regime has generally evolved to reflect the demand for users to capture value. ${ }^{5}$ Passive uses, including scientific uses, have thus been left out, to an extent, of the property regime. The property mismatch approach captures such issues in a property regime.

In our discussion, passive use is akin to a landscape level resource, much like air. Rules that provide for clean air, or in this case, clear spectrum for passive users, can coexist with private property rights, but it is necessary to create frameworks to balance the interests of competing users.

Experience has shown that the initial spectrum property assignment has a great deal of inertia due to its government regulatory nature. As a manufactured resource, substantial capital investments in electromagnetic transducers such as radios, receivers, antennas, etc., are needed to utilize this resource for passive and active users alike. These investments are often highly specific, partly because of physics and partly because of technology. For example, antennas are strongly related to the frequency they are intended to transmit and receive, and technology has limited impact on this. Traditional technology has meant that many components for transmission and reception were hard wired and relatively immutable, although this has substantially changed in recent times with the advent of digital signal processing and software radios. For passive users, the design of receivers with the necessary sensitivity is a costly and time consuming process, and resource limits mean that these users can only change slowly to the more rapidly changing noise environment in which they operate. Passive users may also be constrained by the phenomena they are observing and analyzing.

The creation of private property rights to spectrum has some benefits, including addressing issues with congestion. But the current parceling of the spectrum creates inefficient scales for disparate uses of spectrum resources. One reason, unlike with resource commons, is that technology is always redefining what is possible in terms of performance parameters such as capacity and interference tolerance. Rather than exclusive rights, what could be more appropriate might be a property regime that can be continually adjusted.

The challenge with mismatch differs, as one conventional way to address this is by creating organizations that overcome conflict or have mandates to manage property rights at different scales. Thus, a question may be how to expand the scope of these organizations. We argue that finer virtual parceling of the electromagnetic spectrum could help address the incompleteness of current spectrum license market, allowing a wider and more flexible set of spectrum-usage rights. This can allow better adaptation of communal spectrum usage to random passive phenomenon, and more efficient usage of spectrum in general.

Another possibility is to establish organizations that provide a forum for people to resolve disputes, such as the newly created National Spectrum Innovation Institute, SpectrumX. ${ }^{6}$ Such organizations provide a framework that bring together different users without the presumption that licensing is the most significant way to manage spectrum. The significance of private institutions in governance arises because governments cannot, themselves, do all governing themselves [20]. With spectrum, then, governance includes consideration of the substantive rules, along with the collective choice procedures, with emphasis on how formal authorities interact with private orders (including business, and network associations), to explain change in the rules.

Finally, one consideration with licensing is how much of a role agencies such as the FCC (with commerce in their charter) ought to have, including whether it ought to be abolished [21]. The usual case for abolishing the FCC is that institutions such as the common law would be enough to figure out ownership of spectrum. However, the issue of FCC mismanagement of private property rights to spectrum is not the only reason to reconsider the role of the agency. Another is that it has not historically been equipped to deal with passive use. It is clear from the above analysis that abolishing the FCC might not undermine private property rights to spectrum, and may improve them, but it is far from clear that such an approach would provide for better management of conflicts between active and passive users. In another example of spectrum rights mismatch [22], the FCC's organizational structure is not well suited to dealing with coexistence problems involving mismatched rights. As a result,

${ }^{5}$ This reflects the more general consideration that property rights tend to emerge and change in response to changes in values, as emphasized by [18] and [19].

${ }^{6}$ spectrumx.org 
other institutions may be needed for dealing with these kinds of problems.

\section{ACKNOWLEDGMENT}

We thank Thomas Hazlett for earlier conversations. This work was funded in part by NSF Grant 2037918 and 1642949 as well as the Charles Koch Foundation.

\section{REFERENCES}

[1] T. W. Hazlett, The political spectrum: The tumultuous liberation of wireless technology, from Herbert hoover to the smartphone. New Haven: Yale University Press, 2017.

[2] T. Yandle, "Understanding the consequences of property rights mismatches: A case study of New Zealand's marine resources," Ecol. Soc., vol. 12, no. 2, 2007.

[3] K. Bradshaw, B. Christmas, and D. Lueck, "An introduction to 'overlapping resources and mismatched property rights,'” Int. J. Commons, vol. 14, no. 1, 2020.

[4] M. B. Weiss, W. H. Lehr, A. Acker, and M. M. Gomez, "Socio-technical considerations for spectrum access system (sas) design," in 2015 IEEE International Symposium on Dynamic Spectrum Access Networks (DySPAN), 2015, pp. 35-46.

[5] L. Herzel, "My 1951 color television article," J. Law Econ., vol. 41, no. S2, pp. 523-528, 1998.

[6] R. H. Coase, "The economics of broadcasting and government policy," Am. Econ. Rev., pp. 440-447, 1966.

[7] T. W. Hazlett, "Assigning property rights to radio spectrum users: Why did FCC license auctions take 67 years?," J. Law Econ., vol. 41, no. S2, pp. 529-576, 1998.

[8] P. Bustamante, M. M. Gomez, I. Murtazashvili, and M. B. Weiss, "Spectrum anarchy: why self-governance of the radio spectrum works better than we think," $J$. Institutional Econ., vol. 16, no. 6, pp. 863-882, 2020.

[9] National Research Council, Spectrum management for science in the 21st century. National Academies Press, 2010.

[10] E. Ostrom, Governing the commons: The evolution of institutions for collective action. New York: Cambridge University Press, 1990.

[11] E. Ostrom, Understanding institutional diversity. Princeton: Princeton University Press, 2005.

[12] J. S. Rose, "Telecommunications Policy, Regulation \& Enforcement: A 20 Retrospective of FCC Adjudication," presented at the TPRC48: The 48th Research Conference on Communication, Information and Internet Policy, 2020. doi: https://ssrn.com/abstract $=3749773$ or http://dx.doi.org/10.2139/ssrn.3749773.

[13] O. E. Williamson, "The economics of governance," Am. Econ. Rev. PP, vol. 95, no. 2, pp. 1-18, 2005.
[14] Y. Barzel, A theory of the state: economic rights, legal rights, and the scope of the state. New York: Cambridge University Press, 2002.

[15] J. W. Mayo and S. Wallsten, "Enabling efficient wireless communications: The role of secondary spectrum markets," Inf. Econ. Policy, vol. 22, no. 1, pp. 61-72, 2010.

[16] E. A. Posner and E. G. Weyl, Radical markets: Uprooting capitalism and democracy for a just society. Princeton: Princeton University Press, 2018.

[17] R. H. Coase, "The federal communications commission," J. Law Econ., vol. 2, pp. 1-40, 1959.

[18] A. A. Alchian and H. Demsetz, "The property right paradigm," J. Econ. Hist., vol. 33, no. 1, pp. 16-27, 1973.

[19] H. Demsetz, "Toward a theory of property rights," Am. Econ. Rev., vol. 57, no. 2, pp. 347-359, 1967.

[20] P. D. Aligica, P. J. Boettke, and V. Tarko, Public Governance and the Classical-liberal Perspective: Political Economy Foundations. New York: Oxford University Press, 2019.

[21] T. W. Hazlett, "Optimal abolition of FCC spectrum allocation," J. Econ. Perspect., vol. 22, no. 1, pp. 103$128,2008$.

[22] P. Krishnamurthy, I. Murtazashvili, and M. B. Weiss, "On the Coexistence of Disparate Spectrum Users: A Property Rights Mismatch Approach," presented at the PRC49: The 49th Research Conference on Communication, Information and Internet Policy, 2021. doi: https://papers.ssrn.com/sol3/papers.cfm?abstract_id=38 97993. 